\title{
Preselection of Electronic Services by Given Business Services Based on Semantic Concept Correspondence Applied for the Logistics Domain
}

\author{
Rolf Kluge $e^{1,2}$ \\ ${ }^{1}$ Information Systems Institute, University of Leipzig, \\ Grimmaische Str. 12, 04109 Germany \\ rkluge@wifa.uni-leipzig.de \\ ${ }^{2}$ Department of Computing, Macquarie University, \\ Sydney, NSW 2109, Australia \\ rolf.kluge@mq.edu.au
}

\begin{abstract}
Service oriented environments consist of electronic and business services. Business services encapsulate core business activities whereas electronic services support the operation of business services by means of enterprise software applications. In environments with a large number of business and electronic services, methods for the selection of electronic services for certain business services are needed. This paper presents a conceptual approach for a preselection of electronic services that potentially fits the needs of a given business service based on semantic concept correspondence. The approach is based on the hypothesis that the higher the correspondence between semantically described concepts of business and electronic services the higher the probability of a match between them. This will support decision making during electronic service evaluation. The approach is elaborated based on certain requirements and evaluated by a use case scenario of the logistics domain.
\end{abstract}

Keywords: Service Evaluation, Service Discovery, Preselection, Semantic Web services, Ontology Matching, Logistics, Transportation Management.

\section{Introduction}

Companies are constantly faced with changing market conditions and threats, new competitive pressures in terms of cost, time, and flexibility, and ever changing regulations that require compliance. In order to cope with those conditions, companies outsource internal applications to external service providers in order to focus on the growth of their core activities and competencies. This holds particularly true in the area of logistics. Outsourcing includes business functions such as warehousing, transportation, transshipment, order management, etc., but also computing functions such as enterprise software applications. Furthermore, service providers e.g. third and fourth party logistics ${ }^{1}$ engage subcontractors in order to fulfill certain functions. This

1 "A fourth-party logistics provider is an independent, singularly accountable, non-asset based integrator of a clients supply and demand chains." [1]. 
leads to new markets and business models for providing business and computing functions as well.

With the advent of service orientation an important design paradigm that significantly eases outsourcing was established. Service orientation utilizes services as basic abstractions of business and computing functions. Services are defined as selfcontained, loosely coupled entities that encapsulate a limited piece of functionality. Services are reusable, are able to be composed, and they provide a well defined external interface. Service orientation as a design paradigm for business and computing functions lets enterprises easily integrate externally provided services into internal processes and promises a number of benefits - among them flexible re-configuration, dynamic binding, easy access to heterogeneous resources and processes, transparency across implementation details and last but not least a relatively stable set of standards for aspects such as interface, orchestration, choreography or contracting.

Research on service orientation has been conducted in various directions. One of them is the application of the service oriented design paradigm towards business concerns and capabilities by means of business services (BS) [2] and towards encapsulating computing systems, information systems and software applications by means of electronic services (ES) [3]. An area which received insufficient support so far is the alignment of business and electronic services. Business and electronic services are naturally interrelated as ES provide the fundamental support by means of data, information and processing. However, in environments with a high number of BS and also usually a high number of ES that can be bound to support these BS the decision on which ES candidate provides the most suitable support for a certain BS is not trivial.

In order to provide decision support in the context of service evaluation, this paper presents a conceptual approach for a preselection of ES for given BS based on the semantic correspondence of concepts. The approach is based on the hypothesis that the higher the correspondence between semantically described concepts the higher the match between a business and an electronic service. The paper presents requirements, derives basic constituents and illustrates the approach by an example in the area of logistics.

The remaining of this paper is structured as follows: First of all, requirements as well as the approach and its constituents are described in chapter 2. Basically, the approach contains the three building block models, and preselection logic. These building blocks are examined in detail in chapter 3. Section 4 shows related work and chapter 5 draws a conclusion and mentions future work.

\section{Requirements and Conceptual Approach}

For preselection of ESs for given BSs based on their semantic concept correspondence a number of generic requirements must be satisfied. These requirements are described below and will be used as the basis for deriving the constituents of the approach. Conceptualization \& formalization: Calculating of semantic concept correspondence of subjects such as business and electronic services requires that relevant aspects of them are represented explicitly or implicitly. Comparison: On the basis of formal conceptualizations, a general mean to compare both subjects is necessary. Thus, conceptualizations and their formalization of business and electronic services 
need to be aligned to each other in a way that some matching logic can conclude about their semantic correspondence level. Quantification: Once conceptualizations of business and electronic services are aligned to each other by means of comparability, processing logic that is able to determine a semantic correspondence level between business and electronic services must be applied. Aggregation: Since the number of subject conceptualization entities may be high and lead to multi-dimensional vectors, aggregation logic that consolidates correspondence levels on a concept level towards service level correspondence is needed. Visualization: The results of determining correspondence levels for a large number of business and electronic service subjects on a service-level may still be complex and due to its numeric and multi-dimensional character difficult to interpret by humans. Thus, a visualization metaphor for representation of large scale correspondence levels and formatting them towards easy interpretation by humans for decision making is required. (Semi-)Automation: Since the selection and evaluation of electronic services for a certain business service can be fulfilled manually as well, the approach should facilitate automation in order to gain cost and time efficiency. However, since full automation may lead to error-prone results the approach should leave space for manual steps.

The constituents of the approach for the preselection of ESs for given BSs due their business semantics are derived from requirements described above. The conceptual approach is illustrated by Fig. 1.

For formalization, conceptualization and comparison reasons a service meta-model (called service comparison meta-model SCMM) is needed. The SCMM contains for example underlying processes, resources, capabilities, etc. Since there are several service models preexisting (cf. 0. Related Work), the elements of the SCMM can be derived from them. However, the SCMM must fulfill the following requirements: First, the meta-model has to be commonly applicable for BS and ES in order to satisfy basic comparability. Second, basic service elements, such as capabilities, processes, resources, etc., and aspects such as internal and external, static and dynamic characteristics have to be considered. Third, the SCMM must leave space for semantic variation.

The SCMM is applied to several BSs and ESs, which results in several Business Service Comparison Models (BSCM) $\mathrm{x}_{\mathrm{i}}$ and Electronic Service Comparison Models (ESCM) $y_{j}$. Each BSCM $x_{i}$ contains many BS Concepts $n_{i, k}$, whereas $i$ is the index of the $\mathrm{BS}$ and $\mathrm{k}$ is the index of the concept. Analogously, each ESCM $\mathrm{y}_{\mathrm{j}}$ contains many ES Concepts $\mathrm{m}_{\mathrm{j}, \mathrm{l}}$, whereas $\mathrm{j}$ is the index of the ES and 1 is the index of the concept. These concepts have to be extracted, normalized and formally described for further processing.

Next, matching logic can be applied in order to conclude about the correspondence level for each $n_{i, k}-m_{j, 1}$-combination. The application of the matching logic results in a correspondence value $c_{i, j, k, l}$. The correspondence value is a quantification of the similarity between a certain BS concept $n_{i, k}$ and a certain ES concept $m_{j, 1}$. Since there are many $(\mathrm{k} * \mathrm{l})$ possible concept combinations for each BS-ES-combination $(\mathrm{i} * \mathrm{j})$ automation is needed. Automation is expressed by the function $f\left(n_{i, k}, m_{j, 1}\right)$. After having similarity measures on semantic concept level, the values have to be aggregated for a certain BS-ES combination in order to conclude about similarity on service-level and service-element-level. The aggregation is embodied by the function $\mathrm{f}\left(\mathrm{c}_{\mathrm{i}, \mathrm{j}, 1,1}, \mathrm{c}_{\mathrm{i}, \mathrm{j}, 1,2}, \ldots\right.$, $\mathrm{c}_{\mathrm{i}, \mathrm{j}, \mathrm{k}, \mathrm{l}}, \ldots \mathrm{c}_{\mathrm{i}, \mathrm{j}, \mathrm{K}, \mathrm{L}}$ ) taking into account each $\mathrm{c}_{\mathrm{i}, \mathrm{j}, \mathrm{k}, \mathrm{l}}$ value. There is a quantifiable similarity value for each combination $\mathrm{s}_{\mathrm{i}, \mathrm{j}}$ as a result of the aggregation. 


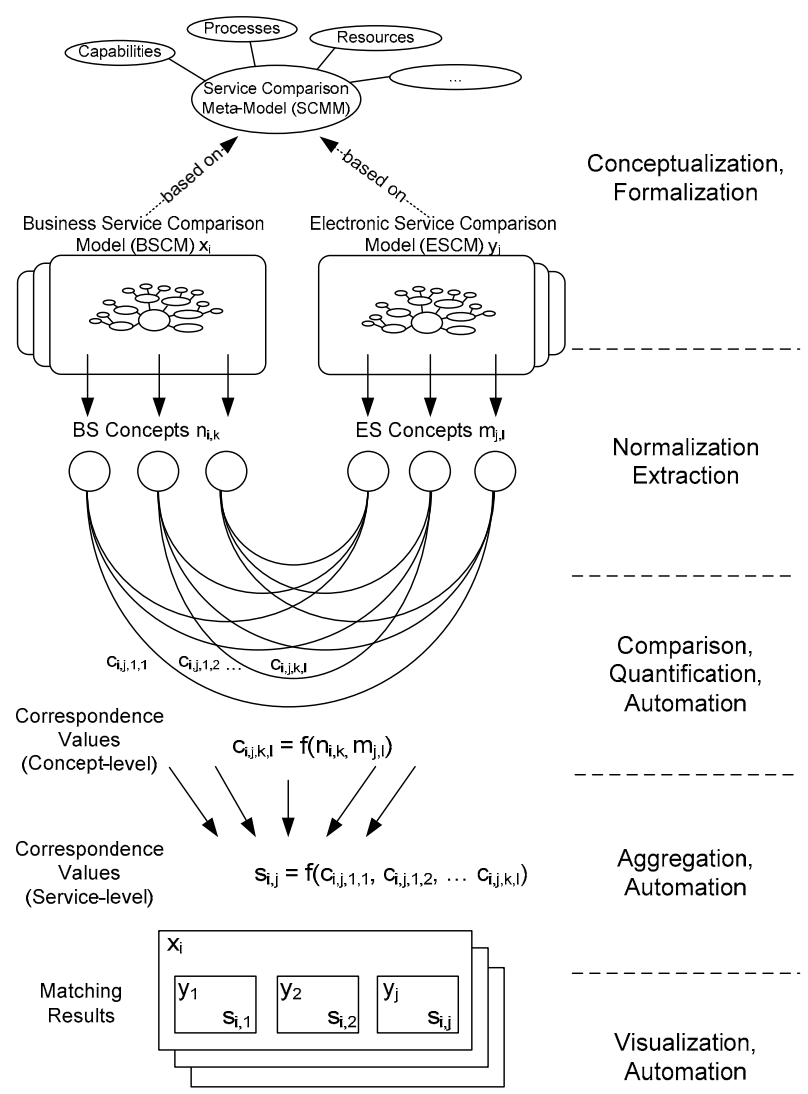

Fig. 1. Conceptual Approach for Preselection

For achieving the goal of supporting decision making the pure values are not sufficient. They have to be represented and visualized. This is depicted by the ranked list of high potential ESs for each BS in the lower part of Fig. 1.

\section{Constituents in Detail}

The conceptual approach the building blocks: SCMM as well as its instantiations (i.e. BSCM and ESCM), and preselection logic. These are examined in detail below. Visualization exceeds the scope of this paper.

\subsection{Service Comparison Meta-model}

A meta-model describes the structure of a possible model including constructs, relationships between them as well as restrictions and modeling rules. Furthermore, a meta-model describes the structure in an abstract way. The concrete syntax of that is provided by the modeling-language. Whereas the abstract syntax (i.e. the meta-model) provides the foundation for the automated and tool-supported processing of a model, 
the concrete syntax provides concrete constructs in a textual or graphical manner that is used for modeling [4]. Adapting these definitions for the preselection approach, the meta-model defines common elements for comparison. As already mentioned, the meta-model is called service comparison meta-model (SCMM). Instantiations of the SCMM lead to several BS- and ES-models, which describe each service partly, but contain only comparable aspects. The BS-model is called Business Service Comparison Model (BSCM) and the ES-model is called Electronic Service Comparison Model (ESCM). For representation purposes a modeling-language will make BCSM and ECSM manageable. Since modeling-language as well as the BSCM and ESCM have to be in accordance to the SCMM and since such a SCMM does not exist so far, the elements of the SCMM has to be elaborated. There are several existing approaches for service modeling (cf. 0. Related Work). Thus, the SCMM can be derived from them in accordance to the three already mentioned SCMM requirements (cf. chapter 3). However, since the conceptual approach is widely independent from the underlying SCMM and the fact that a SCMM is hard to predefine, because there are always alternatives, we provide a very generic and simplistic meta-model for this document. The SCMM is derived from [5]. Karakostas and Zorgios define that a service contains capabilities, processes, and resources. A resource is either a tangible (e.g. a truck of a logistics company) or an intangible (e.g. transport order information). Resources are governed by the service provider and must be in place in order to fulfill a service. A process coordinates resources, is created and managed by the service provider, and issues capabilities. A capability embodies the functionality of the service (cf. [5]). These service elements supplemented by actions, which are atomic operations of a process, including their relationships, leads to the SCMM. For simplification purposes services, capabilities, actions and resources are characterized by their name and a description. The process, which comprises actions and resources, is described by a process description as a BPMN diagram [6]. The textual expression of name and description as well as the BPMN diagram and its graphical representation form the modeling-language of the SCMM.

An example for a BS is a standard transportation service fulfilled by a freight forwarding company. The result of the BSCM by using the SCMM is depicted on the left hand side of Table 1 . The service is a truck cargo transport. The capability of the service is that the company fulfills all actions from transport planning to transport execution. In detail, the actions are plan transport order, load cargo, transport cargo physically, and unload cargo at destination. The process is depicted as a BPMN. The process has a start as well as an end event and contains all mentioned actions. Further, the process contains resources as inputs and outputs of actions. Resources are transport order, transport unit capacity, driver capacity, shipment document and the delivery bill. On the other hand, there is an example for an ES taken from the SAP® Transportation Management module "Planning and Optimization". Information about SAP® Transportation Management is gained from the user interface of the software and from literature [7]. This results in the ESCM illustrated on the right hand side of Table 1. The ES is a tour planning service, which supports the planning of cargo transport tours. The service has the capability to plan and optimize tours, which means that certain transport orders are allocated to certain transport units and the optimal route, i.e. the optimal sequence of transport locations, is calculated. The service comprises two actions - clustering and routing. Clustering comprises the 
transport order planning, the tour planning, and engaged vehicle and driver planning. The service process contains both actions and some information resources as input and output parameters. The resources are optimization parameters, restrictions, master data, tour list, and route scheduling. Each resource element has sub-resource elements as shown in the description.

Table 1. BSCM and ESCM example

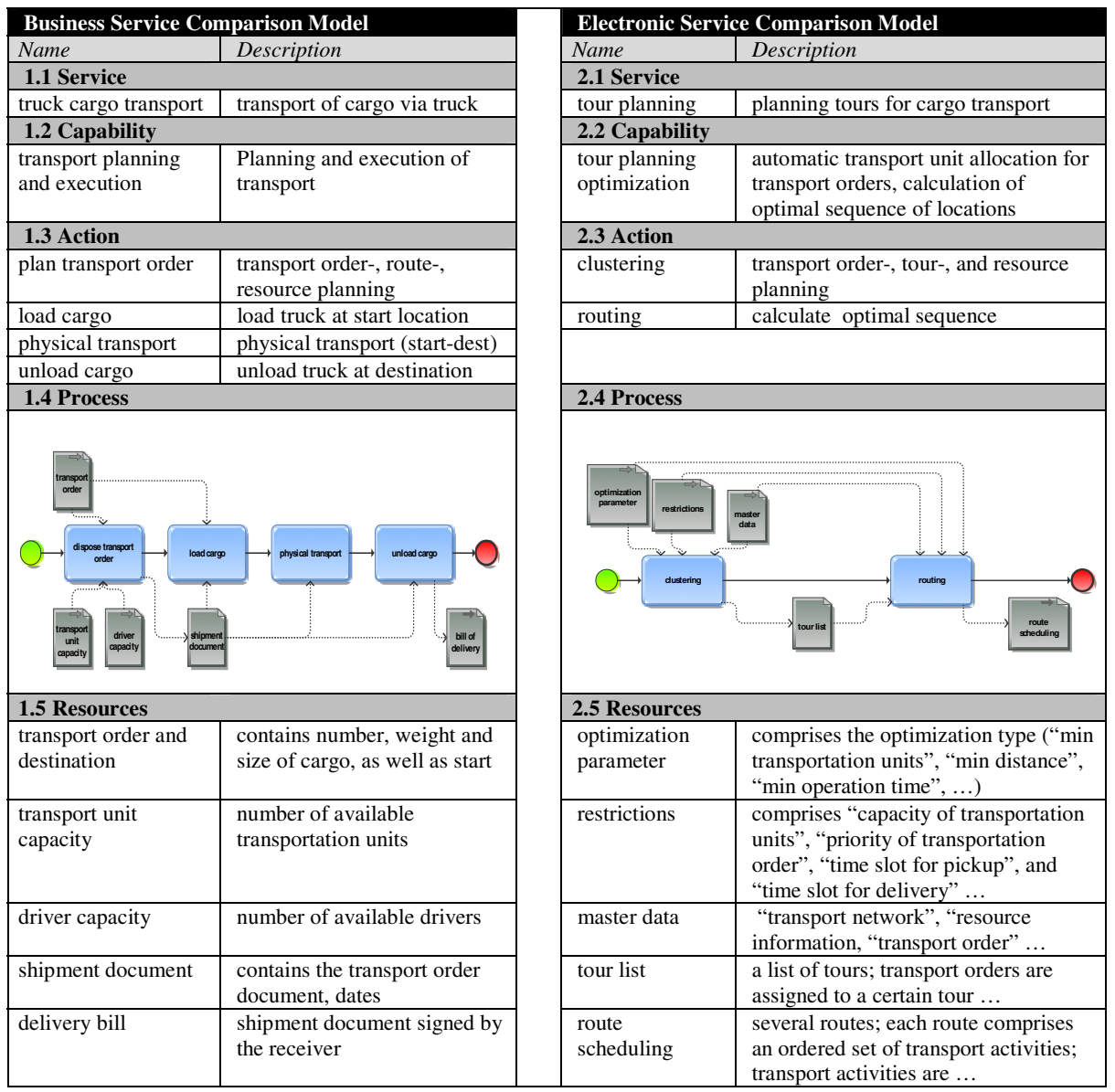

\subsection{Preselection Logic}

Based on BSCM and ESCM descriptions, preselection logic can be applied in order to measure the terminological correspondence between BS and ES. Formalization, normalization and extraction of concepts are necessary in order to make BSCM and ESCM descriptions machine processable, first. Second, comparison logic will be applied, in order to measure correspondence for each single concept. Third, 
aggregation logic will be applied in order to measure the correspondence on element level, i.e. elements that are defined in the SCMM.

The description of BS and ES depicted in Table 1 are semi-formal and cannot be processed automatically so far. Thus, the BS and ES description has to be transformed into a formal model. For conceptual comparison, it is suitable to use semantic languages, which provide the expression of concepts and their relationships. An appropriated semantic language is OWL DL [8]. The transformation from the service description to a semantic representation of concepts is a straight-forward procedure, since there are Ontologie Engineering methodologies [9] available. However, some special cases have to be handled. Thus, the following rules will be applied for formalization and concept extraction within this document: (1) single nouns are single concepts, (2) compound nouns are split into their parts and treated as single nouns, (3) verbs are normalized as nouns, (4) adjectives and copulas are skipped and (5) each concept appears only once. Taking the five rules into account and applying the METHONTOLOGY [9] results in the semantic formalization is shown below. Fig. 2 shows a representation of the ontology created with Protégé ${ }^{2}$. Regarding the BSCM, Fig. 2 shows the SCMM elements on the left side, including the service element, the capability element, the action element, the resource element and the process element (cf. ellipses with dotted line). Since a process contains resources and actions, the description of a process is the summation of the resource elements description and the action elements description. The description statements are next to the SCMM elements. These are connected as stated in the description itself. Furthermore, there are extracted concepts on the right hand side of the BSCM description statements (cf. ellipses with dashed lines). These concepts are extracted following the five rules mentioned above. Such a OWL DL description exists for the ESCM as well (cf. right hand side of Fig. 2). All in all there are 29 single concepts extracted from the BSCM and 52 concepts extracted from the ESCM.

After the extraction of single concepts, correspondence values between them are measured. Several matching algorithms and strategies for measuring similarity between concepts are presented in literature (cf. 0. Related Work). However, for simplification purposes we chose a basic matching algorithm for this example (similarity 1 if concept names are equal and similarity 0 if concept names are different). Out of 29 single business service concepts there are 16 matches to single electronic service concepts (e.g. cargo, transport, plan, order, location, etc.). Next, single results have to be mapped to their roots in order to reveal matching results on combined conceptual level, service-element level (i.e. capability, process, action, and process), and service level. Thus, single matching results have to be aggregated on a combined conceptual level first. As an example the service description "truck cargo transport" contains three single concepts. Two of them - cargo and transport - match to single electronic service concepts. As an aggregation the combined concept "truck cargo transport" matches to two third $(2 / 3)$ to the electronic service. In a similar way the concept match of levels above is calculated. As an example, the resource "transport order" contains the concept cargo and cargo contains weight and size. Weight matches $0 / 1$, size matches $0 / 1$ as well, cargo matches $1 / 1$, start location matches $2 / 2$, destination location matches $2 / 2$, and transport order itself matches $2 / 2$. The match on transport

${ }^{2}$ http: //protege.stanford.edu/ 


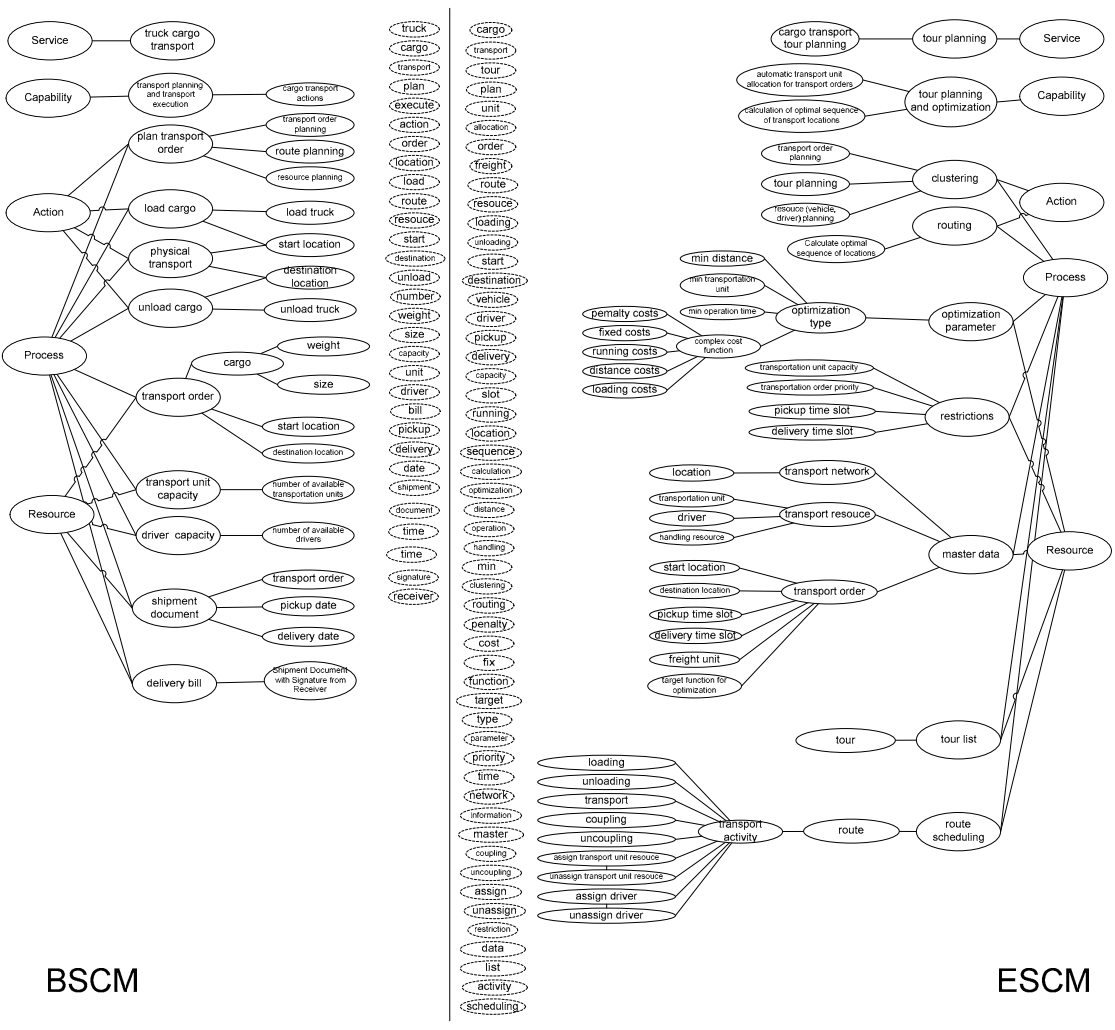

Fig. 2. BSCM and ESCM example as OWL

Table 2. Matching results on Service-Element Level

\begin{tabular}{ll}
\hline Element & Matching value \\
\hline Service & 0.6667 \\
Capability & 0.7143 \\
Action & 0.9200 \\
Process & 0.7222 \\
Resource & 0.5517 \\
\hline
\end{tabular}

order level is $0.7778(=0 * 1 / 9+0 * 1 / 9+1 * 1 / 9+1 * 2 / 9+1 * 2 / 9+1 * 2 / 9)$. In the same way a matching on a service-element level is calculated. Results are depicted in Table 2. The aggregated conceptual matching value on a service level is 0.7188 which means that the mentioned business and electronic service match with $71.88 \%$ based on their single concepts (by using this particular procedure). However, the value of $71.88 \%$ as a standalone statement leaves space for interpretation. The question whether the electronic service supports the business service or not is not answered. This can only be answered by applying the approach to several electronic services. Moreover, not only the end result of $71.88 \%$ has to be considered. The sub results on 
service-element levels as well as certain concept matching results are interesting for a detailed evaluation. In order to support interpretation on various levels visualization is needed, though this is not part of this paper.

\section{Related Work}

Electronic Service evaluation and discovery is an important topic in SOA. The SOA basic model addresses this by an own entity called service registry (also known as service broker). A service consumer can discover the registry in order to find a service that fits its concerns (cf. [10]). Basic mechanisms, such as UDDI [11], support the search for Electronic Services based on keywords only. However, there are more sophisticated approaches based on semantic Web service (i.e. ES) description, such as WSMO [12] or OWL-S [13]. Semantic Web service discovery is much more precise, since it is based on the definition of Goals. These Goals have to be defined explicitly in terms of functional and non-functional parameters. However, there are divergent opinions about goals as well, since "service requesters are not expected to have the required background to formalize their goals" [14]. This is true due to the fact that business people (i.e. potential service requestors) know their business, but usually do not know what to expect from a Web service. Further, goals are defined by preconditions, assumptions, postconditions and effects (cf. [12]) using logical languages, but business people are not familiar with logical rules. Moreover, one might think that BSs are similar to Goals, but they are not. Goals define expectations on a (soughtafter) Web service explicitly. In contrast, BSs describe business cases, which might implicitly contain requirements on a ES. With this, the approach for preselection of ES by given BS based on semantic concept correspondence fits in the gap between a keyword based service discovery and service discovery mechanisms based on semantic Web services. The approach is more precise than keyword based discovery, since not only keywords but the terminology of the business in terms of BSs as well as semantic concept correspondence are taken into account. In contrast it is less precise than a sophisticated semantic Web service discovery. However, the approach is reasonable for preselection purposes, i.e. reducing a large number of available ESs to a significant smaller set, which makes evaluation less complex and less expensive. Furthermore, it does not rely on Goals which might be hard to define and which are described in a language business people do not understand. This is similar to [15], where business process models are used for ES discovery. However, [15] relies on a common domain ontology, which is not considered here. Furthermore [15] refers to [16] for calculating matching degrees, but does not provide any matching or aggregation algorithm. More related work can be found within the areas of the three building blocks service comparison meta-model, preselection logic and visualization. For instance, there is related work in service modeling focused on business concerns (e.g. [17]). Most of these have their root in the marketing area. Further, there are service models and frameworks for ES (e.g. [13]) and there are even approaches that promise to be independent from business and technical concerns (e.g. [18]). Concerning preselection logic there are accepted workings for semantic concept extraction (e.g. [9]). Furthermore, there are algorithms for ontology matching as well (cf. [19]). This area provides techniques for quantifying similarity between semantic concepts. There are several matching techniques, which can be divided into element- and structure-level 
techniques at first stage and into syntactic, external and semantic techniques at a second stage. However, single matching techniques are not sufficient. Usually, matching techniques are combined in order to get a proper matching result. There are sequential matching strategies, which put some matching techniques and variations into a sequence, as well as parallel matching strategies, which perform matching techniques at the same time and aggregate results afterwards (cf. [19]). Last not least, there are approaches for visualizing multi-dimensional results, such as heatmap visualization [20].

\section{Conclusions and Future Work}

Business services describe business concerns structured as services according to the service-orientation paradigm. Electronic services describe technical aspects as services (i.e. according to the service-orientation paradigm) with a special focus on enterprise software applications. Electronic services support operations of business services. Assuming there is a large number of business and electronic services, the evaluation of an electronic service that supports a business service best is time and cost consuming. This paper provides an approach which supports the evaluation process. It is based on the hypothesis that a certain electronic service matches a certain business service if electronic and business services are similar in their semantic concepts. The approach provides a procedure for measuring similarity between business and electronic services on conceptual level.

The application of the approach to a certain business service and a certain electronic service shows that there is a variety of possibilities for conceptualization, matching logic and aggregation logic. The example use case from the logistics domain resulted in a $71.88 \%$ conceptual match between a certain (logistics) business and a certain (logistics) electronic service. It is obvious that this value can vary using different aggregation functions, matching algorithms or even different descriptions of the same use case. Thus, it cannot be attested that a higher matching results represents a better combination than a lower matching results in each case, especially when values are very close to each other (e.g. 75\% and 73\%). This is due to the fact that description might be vary or imprecise. However, assuming that all BS and ES description are truly been made and complete, it can be attested that a BS-ES-combination with a higher matching value has higher probability to be a better solution than a BS-ES-combination with a significant lower matching value. Further, with the same settings and the same business service the approach reveals its significance by its application to a large number of electronic services.

Future work will focus the application of the approach by varying service meta-models, conceptualization methods, preselection logic (in terms of matching algorithms, matching strategies and aggregation logic functions), and visualization possibilities. Moreover, since the tool does not exist so far, this will be developed for handling, application, analyzing, as well as result interpretation.

\section{Acknowledgment}

The work presented in this paper was partly funded by the German Federal Ministry of Education and Research under the projects InterLogGrid (BMBF 01IG09010F) and Logistics Service Bus (BMBF 03IP504). 


\section{References}

1. Win, A.: The value a 4PL provider can contribute to an organisation. International Journal of Physical Distribution \& Logistics Management 38(9), 674-684 (2008)

2. Hering, T., Ludwig, A., Franczyk, B.: Proliferation in Service Types: Towards a Unifying Taxonomy of Service Types. In: International Symposium on Services Science, Leipzig (2009)

3. Papazoglou, M.P., Georgakopoulos, D.: Service-oriented computing - Introduction. Communications of the ACM 46(10), 24-28 (2003)

4. Petrasch, R., Meimberg, O.: Model-Driven Architecture: Eine praxisorientierte Einführung in die MDA, vol. 1. Dpunkt Verlag (2006)

5. Karakostas, B., Zorgios, Y.: Engineering Service Oriented Systems: A Model Driven Approach. Idea Group Publishing, USA (2008)

6. Miers, D., White, S.A.: Bpmn Modeling and Reference Guide. Future Strategies Inc. (2008)

7. Lauterbach, B., et al.: Transportation Management with $\mathrm{SAP}^{\circledR} \mathrm{TM}$, vol. 1. Galileo Press, Bonn (2009)

8. Lacy, L.W.: OWL: Representing Information Using the Web Ontology Language. Trafford Publishing, Crewe (2005)

9. Gómez-Pérez, A., Fernández-López, M., Corcho, O.: Ontological Engineering. In: Advanced Information and Knowledge Processing, vol. 1. Springer, Berlin (2004)

10. Papazoglou, M.P.: Web Services: Principles and Technology. Prentice Hall, Essex (2007)

11. Clement, L., et al.: UDDI Version 3.0.2

12. Roman, D., Lausen, H., Keller, U.: Web Service Modeling Ontology - WSMO Final Draft (D2v1.3). DERI, Innsbruck (2006)

13. Martin, D., et al.: OWL-S: Semantic Markup for Web Services, W3C Member Submission

14. Keller, U., et al.: Automatic Location of Services. In: Gómez-Pérez, A., Euzenat, J. (eds.) ESWC 2005. LNCS, vol. 3532, pp. 1-16. Springer, Heidelberg (2005)

15. Markovic, I., Karrenbrock, M.: Semantic web service discovery for business process models. In: Weske, M., Hacid, M.-S., Godart, C. (eds.) WISE Workshops 2007. LNCS, vol. 4832, pp. 272-283. Springer, Heidelberg (2007)

16. Preist, C.: A conceptual architecture for semantic web services. In: McIlraith, S.A., Plexousakis, D., van Harmelen, F. (eds.) ISWC 2004. LNCS, vol. 3298, pp. 395-409. Springer, Heidelberg (2004)

17. Shostack, G.L.: How to Design a Service. European Journal of Marketing 16(1), 49-63 (1993)

18. Nayak, N., Nigam, A.: Modeling Business Services for Implementing on Global Business Services Delivery Platforms. In: 4th IEEE International Conference on Enterprise Computing, E-Commerce and E-Services (CEC-EEE 2007), Tokyo, Japan, pp. 577-583 (2007)

19. Euzenat, J., Shvaiko, P.: Ontology Matching. Springer, Heidelberg (2007)

20. Pryke, A., Mostaghim, S., Nazemi, A.: Heatmap visualization of population based multi objective algorithms. In: Obayashi, S., Deb, K., Poloni, C., Hiroyasu, T., Murata, T. (eds.) EMO 2007. LNCS, vol. 4403, pp. 361-375. Springer, Heidelberg (2007) 